\title{
Expansion of mesenchymal stem cells from human pancreatic ductal epithelium
}

\author{
Karen L Seeberger ${ }^{1}$, Jannette M Dufour ${ }^{1}$, Andrew M James Shapiro ${ }^{1,2}$, Jonathan RT Lakey ${ }^{1,2}$, \\ Ray V Rajotte ${ }^{1,2,3}$ and Gregory S Korbutt ${ }^{1,2}$ \\ ${ }^{1}$ Surgical-Medical Research Institute, University of Alberta, Edmonton, AB, Canada; ${ }^{2}$ Department of Surgery, \\ University of Alberta, Edmonton, AB, Canada and ${ }^{3}$ Department of Medicine, University of Alberta, \\ Edmonton, $A B$, Canada
}

\begin{abstract}
Fibroblast-like cells emerging from cultured human pancreatic endocrine and exocrine tissue have been reported. Although a thorough phenotypic characterization of these cells has not yet been carried out, these cells have been hypothesized to be contaminating fibroblasts, mesenchyme and/or possibly beta-cell progenitors. In this study, we expanded fibroblast-like cells from adult human exocrine pancreas following islet isolation and characterized these cells as mesenchymal stem cells (MSCs) based on their cell surface antigen expression and ability to differentiate into mesoderm. Analysis by flow cytometry demonstrated that pancreatic MSCs express cell surface antigens used to define MSCs isolated from bone marrow such as CD13, CD29, CD44, CD49b, CD54, CD90 and CD105. In addition, utilizing protocols used to differentiate MSCs isolated from other somatic tissues, we successfully differentiated pancreatic MSCs into: (1) osteocytes that stained positive for alkaline phosphatase, collagen, mineralization (calcification) and expressed osteocalcin, (2) adipocytes that contained lipid inclusions and expressed fatty acid binding protein 4 and (3) chondrocytes that expressed aggrecan. We also demonstrated that pancreatic MSCs are multipotent and capable of deriving cells of endodermal origin. Pancreatic MSCs were differentiated into hepatocytes that stained positive for human serum albumin and expressed endoderm and liver-specific genes such as GATA 4 and tyrosine aminotransferase. In addition, preliminary protocols used to differentiate these cells into insulin-producing cells resulted in the expression of genes necessary for islet and beta-cell development such as Pax4 and neurogenin 3. Therefore, multipotent MSCs residing within the adult exocrine pancreas could represent a progenitor cell, which when further manipulated could result in the production of functional islet beta-cells. Laboratory Investigation (2006) 86, 141-153. doi:10.1038/labinvest.3700377; published online 9 January 2006
\end{abstract}

Keywords: differentiation; endoderm; islet progenitor; mesenchymal stem cell; multipotent

Currently, there is an inherent shortage of cadavaric donors necessary to meet organ and tissue transplantation requirements in all disciplines, particularly in the area of clinical islet transplantation. Based on the Edmonton protocol, as many as two to three donor pancreases are required to obtain sufficient islets for a single type I diabetic patient to become insulin independent. ${ }^{1,2}$

In order to address the shortage of islets for clinical transplantation, research is currently directed towards identifying the elusive pancreatic betacell progenitor. Possible progenitors have been hypothesized to reside within the pancreatic ductal

Correspondence: Dr GS Korbutt, PhD, Surgical-Medical Research Institute, 1074 Dentistry/Pharmacy Centre, University of Alberta, Edmonton, AB, Canada T6G 2N8.

E-mail: korbutt@ualberta.ca

Received 27 July 2005; revised 8 November 2005; accepted 10

November 2005; published online 9 January 2006 epithelium,,$^{3-6}$ acinar tissue and/or pancreatic small cells. ${ }^{7,8}$ Although transplantation of islet grafts containing higher numbers of ductal-epithelial cells results in better clinical outcome, ${ }^{9}$ several in vitro attempts to isolate beta-cell progenitors thought to reside within the human ductal epithelium have been inconsistent. ${ }^{3,6,10,11}$ Recently, we have described an in vitro method that enriches the ductal-epithelial cell fraction while depleting the acinar component of the pancreatic exocrine tissue following human islet purification. ${ }^{12}$ However, in our culture conditions, we had been unable to expand insulin-producing cells from this enriched ductal cell fraction. Interestingly, we have observed an outgrowth of adherent 'fibroblast-like', spindleshaped cells from the ductal-epithelial cell aggregates, which we characterize in the current study as mesenchymal stem cells (MSCs).

MSCs have been derived from a number of human somatic tissues such as bone marrow (BM), adipose, 
dermis, muscle, peripheral blood and compact bone. ${ }^{13-16}$ In vitro isolation and characterization of MSCs is based on their adherence, rapid expansion in serum-containing medium, expression of specific cell surface antigens as well as their ability to differentiate into various mesodermal tissues such as fat, bone, cartilage and muscle. ${ }^{15-18}$ MSCs have also been reported to cross the mesodermal lineage and give rise to endoderm both in vitro and in vivo, ${ }^{18-20}$ and when implanted at a site of injury or systemically delivered in experimental animal models, they demonstrate the ability to migrate to the injury site, initiate tissue repair and restore function. ${ }^{18,21,22}$ We hypothesize that since MSCs are capable of deriving endoderm, ${ }^{19,20,22,23}$ they could be directed to become beta-cells and potentially represent an abundant source of insulin-producing cells for transplantation.

By adopting methodology that is currently used to characterize MSCs isolated from various human adult tissues, we report the isolation and characterization of MSCs from the adult human pancreas. In this study, we demonstrate that pancreatic-derived MSCs express cell surface antigens reported to be present on MSCs isolated from various somatic tissues and show that pancreatic MSCs can be differentiated into mesoderm (bone, adipose and cartilage) in vitro. In addition, we also demonstrate that pancreatic MSCs are multipotent, and capable of in vitro differentiation into endoderm (hepatocytes and pancreatic endocrine cells).

\section{Materials and methods}

\section{Pancreatic Ductal Cell Isolation}

Human pancreatic digests from three independent adult donors ranging from 29 to 58 years of age were obtained following islet purification and cultured as described previously. ${ }^{12}$ Briefly, the islet-depleted tissue fraction (89\% exocrine, $20 \%$ ductal epithelium, $2 \%$ beta-cells) is cultured for 4 days in RPMI 1640 (Roswell Park Memorial Institute) medium (Invitrogen, Burlington, Canada), supplemented with $10 \%$ fetal calf serum (FCS) (Sigma-Aldrich, Oakville, Canada), at $37^{\circ} \mathrm{C}$ in $5 \% \mathrm{CO}_{2}, 95 \%$ air in order to produce cellular aggregates composed primarily of ductal cell epithelium staining greater than $80 \%$ for the ductal-epithelial cell markers, cytokeratin 19 (CK19) and cytokeratin 7 (CK7). The resulting cellular aggregates were then used to derive and expand MSCs.

\section{MSC Expansion and Differentiation}

For expansion of MSCs, pancreatic ductal cell aggregates (day 0) were transferred to $100 \mathrm{~mm}$ tissue culture-treated plates (Fisher Scientific, Nepean, Canada) at an average cell density of 8.0 cellular aggregates $/ \mathrm{cm}^{2}$, then cultured at $37^{\circ} \mathrm{C}$ in $5 \% \mathrm{CO}_{2}$,
95\% air in expansion medium consisting of RPMI 1640, supplemented with $20 \mathrm{ng} / \mathrm{ml}$ each of basic fibroblast growth factor (bFGF) (Invitrogen) and epidermal growth factor (EGF) (Invitrogen), $10 \mathrm{mM}$ HEPES (Invitrogen), $1 \mathrm{mM}$ sodium pyruvate (Invitrogen), 10\% FCS, $100 \mathrm{U}$ penicillin, $1000 \mathrm{U}$ streptomycin (Invitrogen) and $71.5 \mu \mathrm{M}$ beta-mercaptoethanol (Sigma). Once adherent fibroblast-like cells migrated out from the ductal cell aggregates and confluency greater than $80 \%$ (14 days) was obtained, the monolayer was detached with $0.25 \%$ trypsin-EDTA (Invitrogen). Cells were then counted and replated at a density of 2500 cells $/ \mathrm{cm}^{2}$ for up to 13 passages (98 days) in the same culture conditions. For mesoderm differentiation, pancreatic MSCs from passage(s) 2-12 $(n=3)$ were differentiated into bone, fat and cartilage using protocols previously reported for the differentiation of MSCs isolated from $\mathrm{BM}$, adipose and cartilage tissues. ${ }^{16,24}$ For endoderm differentiation $(n=3)$, pancreatic MSCs from passage(s) 2-6 were differentiated into hepatocytes using a multistep protocol described by Lee et al. ${ }^{19}$ For pancreatic endocrine differentiation $(n=3)$, we describe a novel multistep protocol.

For osteogenic differentiation, pancreatic MSCs were allowed to become $80 \%$ confluent and treated with osteogenic-selective medium (Stem Cell Technologies, Vancouver, Canada) according to the manufacture's protocol. The medium was replaced every 3-4 days for 20-31 days. To achieve adipogenic differentiation, pancreatic MSCs were allowed to become $90 \%$ confluent and treated with adipogenic-selective medium (Stem Cell Technologies). The medium was replaced every 3-4 days for 14-20 days. For chondrogenic differentiation, pancreatic MSCs were transferred to a sterile $15 \mathrm{ml}$ conical tube (Fisher Scientific), centrifuged at $200 \mathrm{~g}$ for $5 \mathrm{~min}$ and washed twice with phosphate-buffered saline (PBS). Pancreatic MSC pellets were resuspended in Chondrogenic Differentiation Medium (R\&D Systems Inc., Minneapolis, MN, USA), centrifuged and cultured in the $15 \mathrm{ml}$ conical for 21-28 days with medium changes every 3-4 days. Negative controls consisted of expanded pancreatic MSCs from the same passage number, cultured in expansion medium and not treated with osteogenic, adipogenic or chondrogenic medium.

For hepatogenic differentiation, pancreatic MSCs from passage(s) 2-6 were allowed to become $100 \%$ confluent and differentiated using a three-step protocol (recently described by Lee et $a l^{19}$ ) for differentiating human MSCs isolated from umbilical cord blood and BM. In step 1, the cell monolayer was treated with serum-free IMDM (Iscove's modified Dulbecco's medium) (Invitrogen), supplemented with $20 \mathrm{ng} / \mathrm{ml}$ of EGF and $10 \mathrm{ng} / \mathrm{ml} \mathrm{bFGF}$ for 2 days. In step 2 , the medium was replaced with IMDM, supplemented with $20 \mathrm{ng} / \mathrm{ml}$ of hepatocyte growth factor (Chemicon International Inc., Temecula, CA, USA), $10 \mathrm{ng} / \mathrm{ml} \mathrm{bFGF}$ and $5 \mathrm{mM}$ nicotinamide (Sigma) for 1 week with medium 
changes every 2-3 days. In step 3, IMDM was supplemented with $20 \mathrm{ng} / \mathrm{ml}$ of oncostatin-M (Chemicon), $1 \mu \mathrm{M}$ dexamethasone (Stem Cell Technologies) and $50 \mathrm{mg} / \mathrm{ml}$ ITS (insulin, transferrin, selenous acid) + Premix (BD Biosciences, Mississauga, Canada) for $2-5$ weeks with medium changes every $2-3$ days.

For endocrine differentiation, expanded pancreatic MSCs from passage(s) 2-6 were allowed to become $100 \%$ confluent and differentiated using a three-step, 9-day protocol followed by cellular aggregation in serum-free medium. In step 1, the cell monolayer was treated for 3 days with high glucose DMEM (Dulbecco's modified Eagle's medium) (Invitrogen) supplemented with 1\% FCS. In step 2, the medium was switched to low glucose DMEM (Invitrogen), supplemented with 1\% FCS and $10 \mathrm{mM}$ nicotinamide for 3 days. In step 3, the low glucose medium was supplemented with $1 \%$ FCS, $10 \mathrm{mM}$ nicotinamide and $10 \mathrm{nM}$ exendin-4 (Sigma) for 3 days. After 9 days of treatment, the cell monolayer was detached with $0.25 \%$ trypsinEDTA. Cells were aggregated by reconstituting cells at $400000 \mathrm{cells} / \mathrm{ml}$ in low glucose medium, supplemented with $10 \mathrm{nM}$ exendin-4, $10 \mathrm{mM}$ nicotinamide and $10 \mathrm{ng} / \mathrm{ml}$ transforming growth factor beta-1 (R\&D Systems Inc.) and transferred to a nontissue culture-treated plate for $72 \mathrm{~h}$.

\section{Immunohistochemistry (IHC) and Histology}

Intact ductal cell aggregates, cell suspensions from each passage and differentiated tissue were fixed in Z-fix (Anatech Ltd, Battle Creek, MI, USA), and embedded in $2 \%$ low melting point agarose (Sigma). Once the agar had solidified, the agar blocks were paraffin embedded, cut into $3 \mu \mathrm{m}$ sections and immunostained as described previously. ${ }^{12}$ Primary antibodies were 1/50 CK19 (Dako Cytomation, Mississauga, Canada), 1/1000 insulin (Dako), 1/ 5000 glucagon (Cedarlane Laboratory Ltd, Hornby, Canada), 1/100 amylase (Sigma), 1/1000 human serum albumin (Cedarlane) and 1/50 GATA 4 (Cedarlane). Positive controls were sections of human pancreas and hepatocytes. Negative controls were sections incubated without primary antibody. IHC of osteocytes, adipocytes and chondrocytes was assessed as per the manufacturer's protocol, with primary antibodies to $1 / 200$ osteocalcin (R\&D), 1/ 200 fatty acid binding protein 4 (FABP4; R\&D) and $1 / 200$ aggrecan (R\&D), which are included in the Human Mesenchymal Stem Cell Functional Identification Kit (R\&D).

For histological assessment, differentiated and control tissues were either snap-frozen in Cryomatrix embedding compound (Thermo Shandon, Pittsburgh, PA, USA) and $5 \mu \mathrm{m}$ cryosections were prepared or stained directly on the tissue culture surface. Prior to staining, tissue sections were fixed with $1 \%$ formaldehyde (BDH Laboratory Supplies, UK), except those sections stained for Oil Red O
(ORO). Von Kossa staining ${ }^{25}$ was used to detect mineralization (calcification) of osteocytes. Tissue sections were washed three times in PBS, immersed in water, then immersed in $2 \%$ silver nitrate (Sigma) and exposed to ultraviolet light for $45 \mathrm{~min}$. The slides were then rinsed in water, followed by $5 \%$ sodium thiosulfate (Fisher Scientific) for $3 \mathrm{~min}$ and washed in running tap water before counterstained with Zymed Hematoxylin (Zymed Laboratories, San Francisco, CA, USA). Vector Red Alkaline Phosphatase Substrate Kit (Vector Laboratories, Burlingame, CA) was used to detect alkaline phosphatase (AP) activity of osteocytes. Tissue sections were washed three times in PBS and stained in the dark as per the manufacturer's protocol. Gomori's One-Step Trichrome $\operatorname{stain}^{25}$ was used to detect collagen matrix deposition in bone cultures. Briefly, sections were immersed in water, warmed in Bouin's solution $\left(60^{\circ} \mathrm{C}\right)$ for $30 \mathrm{~min}$, cooled to room temperature in the same Bouin's solution for another $30 \mathrm{~min}$ and washed in warm running tap water. Finally, slides were Trichrome stained for $20 \mathrm{~min}$, then immersed in $0.5 \%$ acetic water. ORO stain was used to detect lipid droplets in adipogenic cultures. ${ }^{25}$ Sections were washed three times in PBS, briefly treated with $70 \%$ ethanol and immersed in $0.4 \%$ ORO (Sigma). Slides were then rinsed with water and counterstained with Zymed Hematoxylin (Zymed Laboratories). Alcian blue stain was used to detect acid mucopolysaccharides ${ }^{25}$ in chondrocytes. After fixation in $1 \%$ formaldehyde, sections were placed in $3 \%$ acetic acid (Fisher Scientific) for $2 \mathrm{~min}$, immersed in 1\% alcian blue (Sigma), $\mathrm{pH} 2.6$, and washed in running tap water.

\section{RNA Isolation and Reverse Transcription-Polymerase Chain Reaction (RT-PCR)}

Ductal cell aggregates from the start of expansion, cell isolates from each passage and differentiated tissue were dissolved in $1 \mathrm{ml}$ of Trizol reagent (Invitrogen) and RNA extracted according to the manufacturer's protocol. cDNA was constructed from $1 \mu \mathrm{g}$ of total RNA using TaqMan Reverse Transcription Reagents (Applied Biosystems, Foster City, CA, USA) according to the manufacturer's protocol and $1 \mu \mathrm{l}$ of cDNA was amplified for 35 cycles using Taq DNA polymerase (Invitrogen). The PCR conditions were as follows: $95^{\circ} \mathrm{C}$ for $30 \mathrm{~s}, 59^{\circ} \mathrm{C}$ for $30 \mathrm{~s}$ and $72^{\circ} \mathrm{C}$ for $30 \mathrm{~s}$ with a final extension of $72^{\circ} \mathrm{C}$ for $8 \mathrm{~min}$ utilizing primer pairs that span at least one intron (Table 1). PCR products were separated on an ethidium bromide-stained (Sigma) agarose gel $(1.5 \%)$ and images captured with Alpha Digidoc software (Perkin-Elmer, Boston, MA, USA). Bands of the expected size were ligated in the pCR5TOPO vector (TOPO TA Cloning Kit for Sequencing, Invitrogen), sequenced (University of Alberta DNA Core Lab) and compared with known GenBank sequences. Positive control consisted of human islet 
Table 1 Primers used for PCR amplification

\begin{tabular}{|c|c|c|c|c|}
\hline Gene & Size $(b p)$ & Strand & Sequence $5^{\prime}-3^{\prime}$ & GenBank \\
\hline \multirow[t]{2}{*}{ Amylase } & \multirow[t]{2}{*}{300} & $\mathrm{~F}$ & GAC TTT CCA GCA GTC CCA TA & \multirow[t]{2}{*}{ NM_020978 } \\
\hline & & $\mathrm{R}$ & GTT TAC TTC CTG CAG GGA AC & \\
\hline \multirow[t]{2}{*}{ Beta-2 microglobulin } & \multirow[t]{2}{*}{268} & $\mathrm{~F}$ & CCA GCA GAG AAT GGA AAG TC & \multirow{2}{*}{ NM_004048 } \\
\hline & & $\mathrm{R}$ & GAT GCT GCT TAC ATG TCT CG & \\
\hline \multirow[t]{2}{*}{ GATA 4} & \multirow[t]{2}{*}{366} & $\mathrm{~F}$ & CTC CTT CAG GCA GTG AGA GCC & \multirow[t]{2}{*}{ NM_002052 } \\
\hline & & $\mathrm{R}$ & GGT CCG TGC AGG AAT TTG ACG & \\
\hline \multirow[t]{2}{*}{ Glucagon } & \multirow[t]{2}{*}{221} & $\mathrm{~F}$ & CCC AAG ATT TTG TGC AGT GGT T & \multirow[t]{2}{*}{ NM_002054 } \\
\hline & & $\mathrm{R}$ & GCG GCC AAG TTC TTC AAC AAT & \\
\hline \multirow[t]{2}{*}{ Insulin } & \multirow[t]{2}{*}{263} & $\mathrm{~F}$ & GCA GCC TTT GTG AAC CAA CA & \multirow[t]{2}{*}{ AY138590 } \\
\hline & & $\mathrm{R}$ & GTT GCA GTA GTT CTC CAG GTG & \\
\hline \multirow[t]{2}{*}{ Ngn3 } & \multirow[t]{2}{*}{313} & $\mathrm{~F}$ & GGT AGA AAG GAT GAC GCC TC & \multirow[t]{2}{*}{ NM_020999 } \\
\hline & & $\mathrm{R}$ & CCG AGT TGA GGT CGT GCA T & \\
\hline \multirow[t]{2}{*}{ NeuroD } & \multirow[t]{2}{*}{439} & $\mathrm{~F}$ & ATC CCA ACC CAC CAC CAA CC & \multirow{2}{*}{ NM_002500 } \\
\hline & & $\mathrm{R}$ & CAG CGG TGC CTG AGA AGA TT & \\
\hline \multirow[t]{2}{*}{ Pax 4} & \multirow[t]{2}{*}{496} & $\mathrm{~F}$ & AGG AGG ACC AGG GAC TAC CGT & \multirow[t]{2}{*}{ NM_006193 } \\
\hline & & $\mathrm{R}$ & TTT AGG TGG GGT GTC ACT CAG & \\
\hline \multirow[t]{2}{*}{ PDX1 } & \multirow[t]{2}{*}{262} & $\mathrm{~F}$ & CCC ATG GAT GAA GTC TAC C & \multirow[t]{2}{*}{ NM_000209 } \\
\hline & & $\mathrm{R}$ & GTC CTC CTC CTT TTT CCA C & \\
\hline Tyrosine & \multirow[t]{2}{*}{358} & $\mathrm{~F}$ & TGA GCA GTC TGT CCA CTG CCT & \multirow[t]{2}{*}{ NM_000353 } \\
\hline Aminotransferase & & $\mathrm{R}$ & ATG TGA ATG AGG AGG ATC TGA G & \\
\hline
\end{tabular}

Table 2 Antibodies for the characterization of cell surface antigens

\begin{tabular}{llll}
\hline Anti-human & Dilution & Fluorochrome & Source \\
\hline CD13 & $3 / 50$ & PE-Cy5 & Caltag Laboratories, Burlingame, CA, USA \\
CD29 & $3 / 50$ & PE-Cy5 & Caltag Laboratories, Burlingame, CA, USA \\
CD34 & $2 / 5$ & PerCP-Cy5.5 & BD Biosciences, Mississauga, CA, USA \\
CD44 & $1 / 10$ & Fluorescein isothiocyanate (FITC) & Santa Cruz Biotechnology, CA, USA \\
CD45 & $3 / 50$ & Phycoerythrin (PE) & Caltag Laboratories, Burlingame, CA, USA \\
CD49b & $1 / 10$ & FITC & Sanquin, Amsterdam, The Netherlands \\
CD54 & $1 / 50$ & FITC & Caltag Laboratories, Burlingame, CA, USA \\
CD90 & $1 / 50$ & PE & BD Biosciences, Mississauga, Canada \\
CD105 & $3 / 50$ & PE & Caltag Laboratories, Burlingame, CA, USA \\
CD117 & $1 / 5$ & FITC & Chemicon International, Temecula, CA, USA
\end{tabular}

or hepatoctye cDNA, while the negative control consisted of water in place of experimental cDNA and beta-2 microglobulin (housekeeping gene) ensured cDNA integrity.

\section{Immunophenotyping by Flow Cytometry}

To determine the phenotype of the cell monolayer, cell surface antigen expression of single cells obtained from each passage was analyzed by flow cytometry. Briefly, cells were fixed with cold $1 \%$ formaldehyde (BDH Laboratory Supplies), washed once with PBS and stained with the primary antibodies summarized in Table 2. Cell populations were analyzed on the FACS Calibur (BD Biosciences, Mississauga, Canada) using Cell Quest Pro software and compared to the isotype control.

\section{Results}

\section{Isolation and Characterization of Cell Preparations}

Human adult pancreatic tissue $(n=3)$ prepared from islet-depleted digests was cultured for 4 days in
RPMI supplemented with 10\% FCS. As reported previously, this procedure results in cellular aggregates $^{12}$ composed primarily of ductal cell epithelium. To isolate MSCs, the ductal cell aggregates (day 0 ) were plated onto tissue culture-treated dishes and cultured with expansion medium. Within 5 days, adherent fibroblast-like cells were observed growing out of the ductal cell aggregates (Figure 1a). Confluency was achieved by day 14 when the cell monolayer was detached by trypsinEDTA treatment and transferred to new $100 \mathrm{~mm}$ tissue culture-treated dishes. After this first passage, the adherent cells divided rapidly and became confluent within 7 days, for a total of 21 days in expansion medium (Figure 1b). The cells were passaged again (passage 2) and cultures were maintained for 13 passages (98 days). During this culture period, at least a 12-fold expansion in cell number was observed between successive passages until passage 9 (70 days) when the fold increase in cell number decreased by $20 \%$. Passage(s) 10-12 had only a five-fold increase, and by passage 13, proliferation had ceased.

The cellular composition of the ductal cell aggregates (day 0) and passaged cells was deter- 

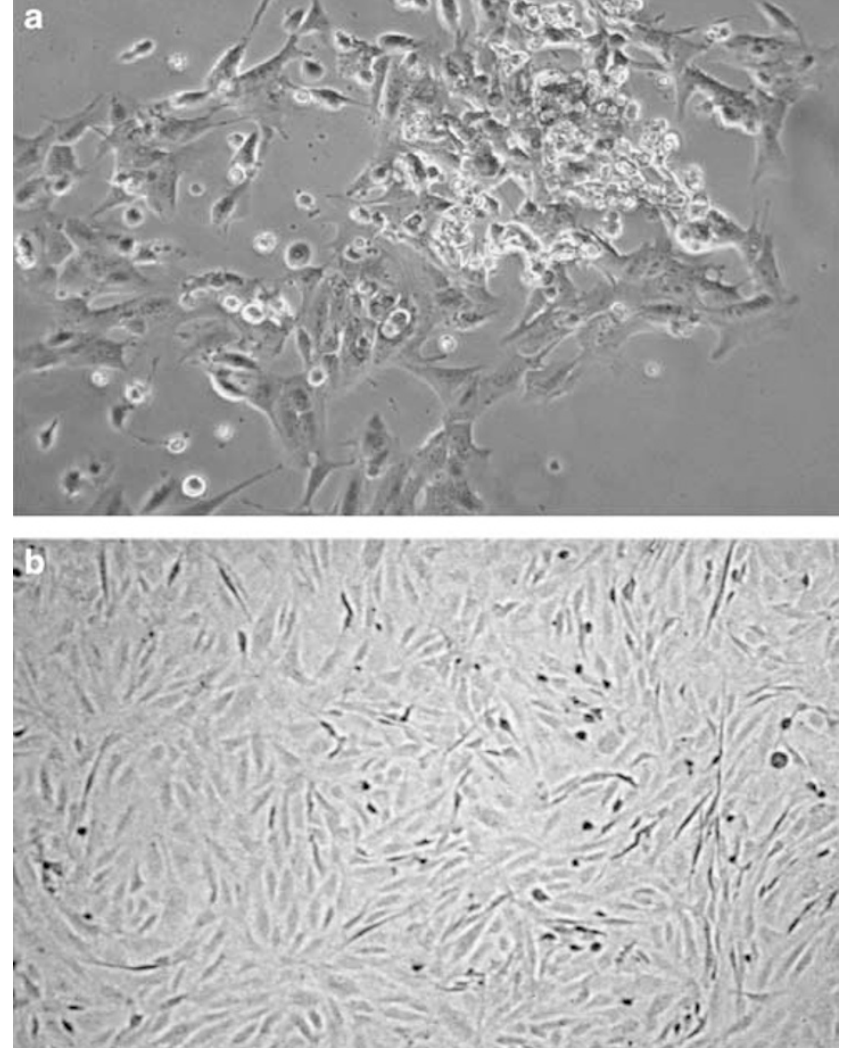

Figure 1 Outgrowth of fibroblast-like cells from pancreatic ductal cell aggregates. After 5 days in expansion medium, adherent, fibroblast-like cells were observed emerging from cultured ductal cell aggregates (a). Magnification $\times 100$. Confluent monolayer of adherent, fibroblast-like cells prior to passage $2(\mathbf{b})$. Magnification $\times 40$.

mined by IHC. At day 0 , the ductal cell aggregates consisted of $81.3 \pm 9.6 \%$ ductal-epithelial cells (CK19), $2.0 \pm 0.9 \%$ beta-cells (insulin), $6.8 \pm 1.1 \%$ acinar cells (amylase) and no alpha-cells (glucagon) were observed (Figure 2a-c). During cellular expansion, immunoreactivity decreased for both exocrine and endocrine markers. Single cells obtained from passage 3 (28 days) consisted of $1.33 \pm 2.3 \%$ CK19, $0 \%$ beta-cells and $1.23 \pm 1.1 \%$ acinar cells (not shown). Single cells from passage 5 (42 days) were negative for CK19, insulin and glucagon staining, but were still comprised of $7.0 \pm 8.0 \%$ acinar tissue (not shown).

Amplification of mRNA confirmed that the fibroblast-like cells expanded from the ductal cell aggregates were not comprised of endocrine cells. Only the initial aggregates (day 0) expressed mRNA for insulin and the insulin transcription factors PDX1 and NeuroD (Figure 3). Although message for glucagon was still present at the time of the first passage (14 days), the mRNA was not translated, since glucagon protein was not detected by IHC. mRNA for amylase was detected in all passages measured correlating with the IHC staining results for amylase. Neither the initial ductal cell aggregates a

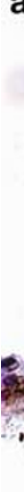

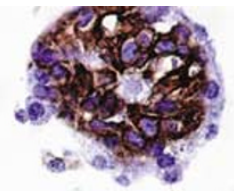
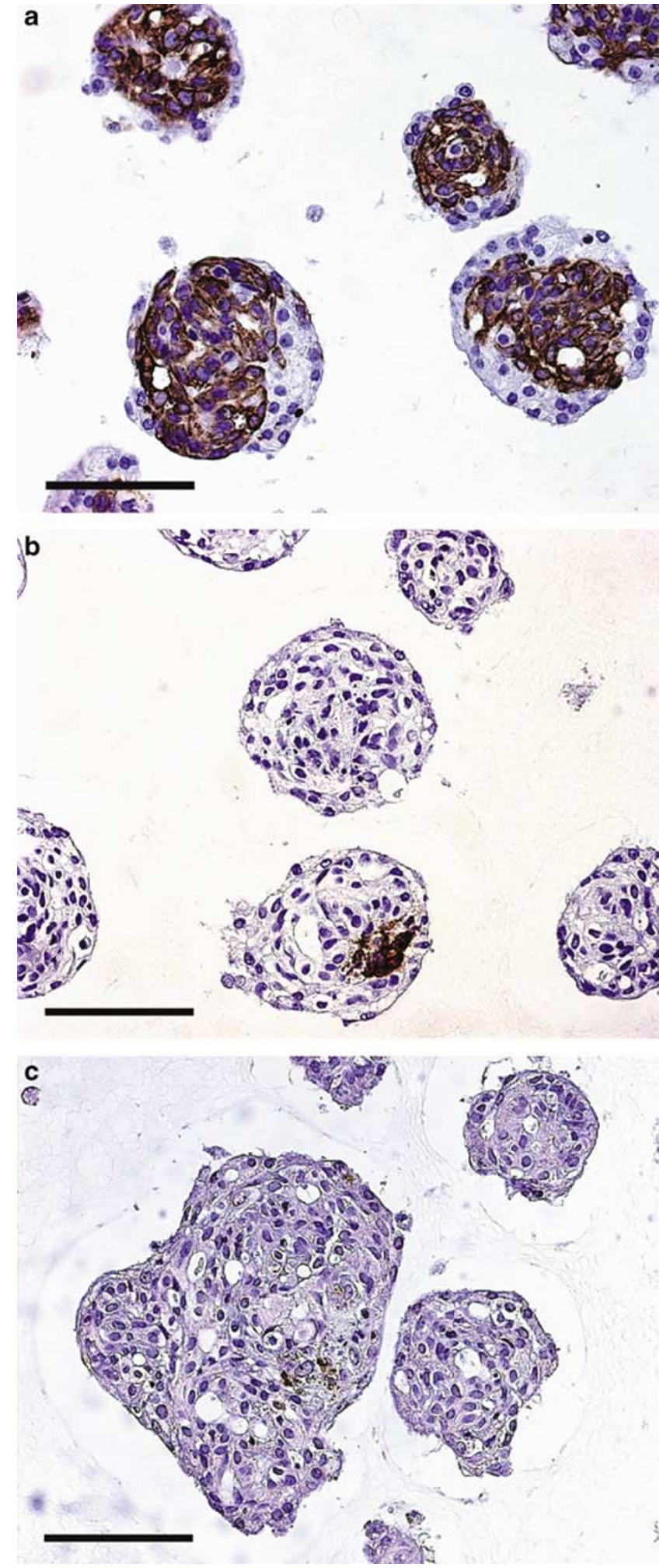

Figure 2 Immunohistochemical staining results of pancreatic ductal-epithelial cell aggregates (day 0) prior to mesenchymal cell expansion. Pancreatic ductal cell aggregates stained positive (a) for the ductal-epithelial cell marker CK19; one aggregate in the field is positive for insulin (b) and amylase (c) staining. Positive immunoperoxidase stain is brown and hematoxylin counterstain is blue. Scale bar represents $50 \mu \mathrm{m}$. 


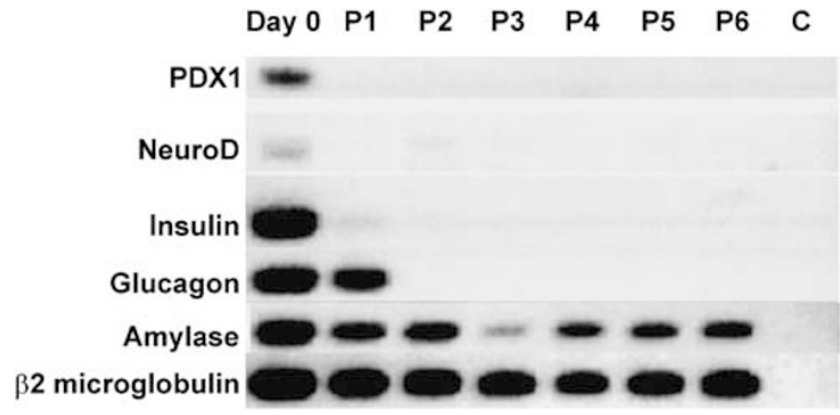

Figure 3 RT-PCR of pancreatic ductal cell aggregates (day 0) and cells from passage(s) 1-6. Message for insulin, and insulin transcription factors PDX1 and NeuroD was only detected in the initial ductal (day 0) tissue and not in subsequent passages, while message for glucagon persists until passage 1 (14 days). Message for amylase is present in all passages shown. Beta-2 microglobulin-positive control denotes RNA and cDNA integrity. Water blank (C) is negative control. (day 0) nor expanded cells expressed mRNA for transcription factors neurogenin 3 (ngn3) or Pax4 (not shown).

Cell surface antigen expression on adherent fibroblast-like cells from all 13 passages $(n=3)$ was analyzed by flow cytometry. Primary antibodies used to describe the phenotype of MSCs isolated from $\mathrm{BM}$, adipose and umbilical cord blood $^{13,16,23,24,26}$ were used in the analysis. Adherent fibroblast-like cells expanded from ductal cell aggregates did not express CD34, CD45and CD117 cell surface antigens, indicating that they were not of hematopoietic origin (Figure 4). These cells did, however, express CD13, CD29, CD44, CD49b, CD54, CD90 and CD105 cell surface antigens, thereby demonstrating an MSC phenotype. This MSC phenotype persisted until passage 13 (98 days). The cells at this time lost their spindle shape,
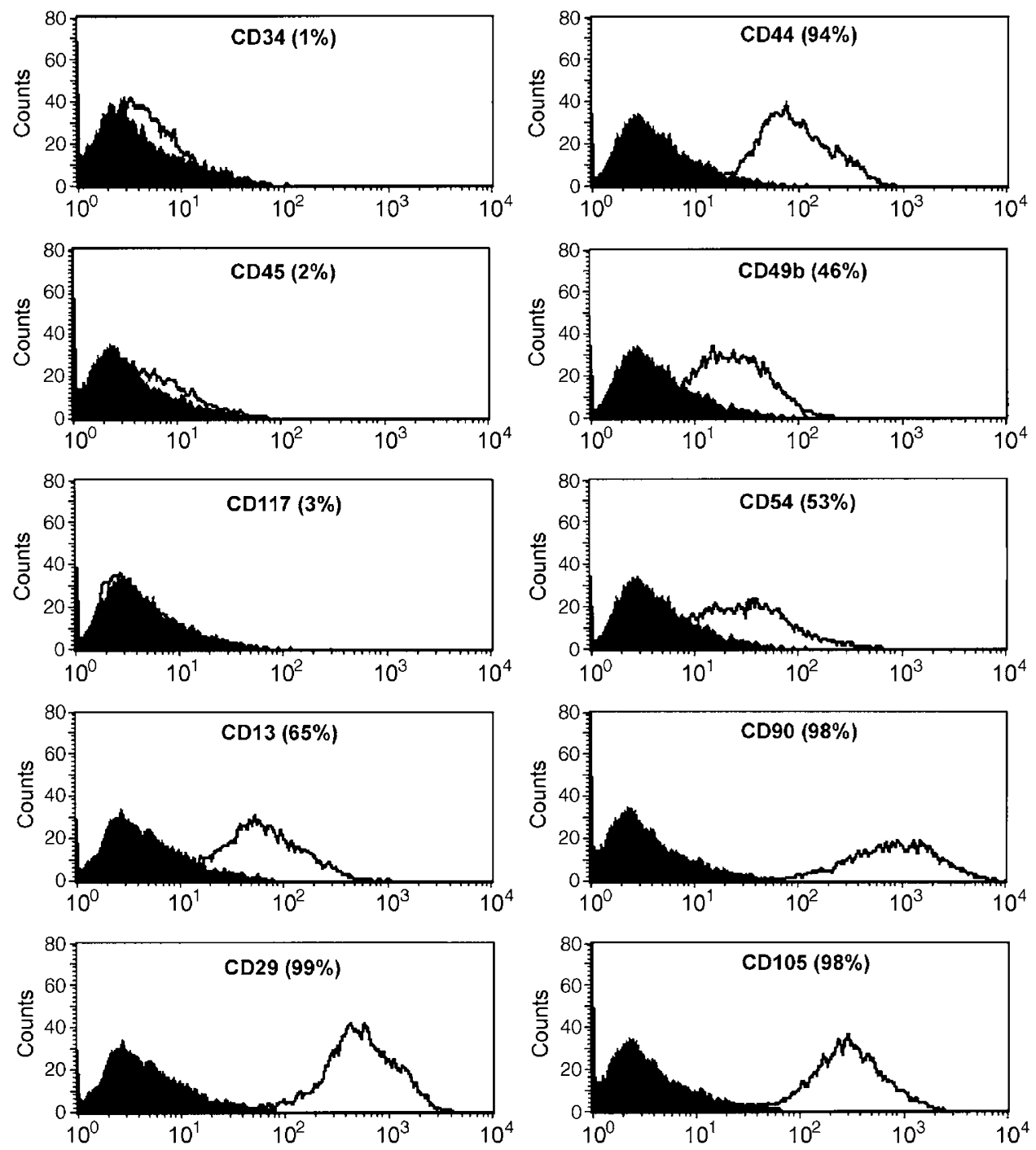

Figure 4 Representative cell surface antigen expression profile of pancreatic mesenchymal stem cells analyzed by flow cytometry. Fibroblast-like cells expanded from the ductal cell aggregates are less than 3\% positive for CD34, CD45 and CD117 and express cell surface antigens CD13, CD29, CD44, CD49b, CD54, CD90 and CD105. Plots show isotype control staining profile (solid) vs specific antibody staining profile (line). 
broadened, lost expression of CD49b and CD13 (not shown) and were no longer capable of proliferation or differentiation.

\section{In Vitro Differentiation of Pancreatic MSCs into Osteocytes, Adipocytes and Chondrocytes}

Osteogenic differentiation was successful when attempted after passage 2 when the cells expressed a predominantly MSC phenotype as demonstrated by flow cytometry (Figure 4). Correspondingly, at passage 13 when these cells no longer expressed an MSC phenotype, differentiation was not possible. During osteogenic differentiation, changes in monolayer cell density were observed after 12 days and in addition to a lattice appearance dense foci were evident within the outer edge of the cell monolayer at 16 days (not shown). After 16 days in osteogenic medium, the monolayer appeared to fold in upon itself and form a single large adherent aggregate (not shown). Tissue harvested and stained at 20 days of differentiation had abundant AP (Figure 5a) and some evidence of mineralization (calcification) demonstrated by Von Kossa staining (Figure 5c). Moderate collagen deposition was demonstrated by Gomori's Trichrome stain (Figure 5e) and IHC for osteocalcin was also positive (Figure 5g). To assess if the developing bone could mature, adherent tissue was treated with osteogenic medium for an additional 11 days. Tissue collected after 31 days of differentiation had scant AP activity (Figure 5b), a substantial amount of mineralization (Figure 5d) and was composed entirely of collagen (Figure 5f). IHC for osteocalcin was also positive (Figure 5h). Undifferentiated controls had slight AP activity, no evidence of mineralization, collagen or osteocalcin expression (not shown).

Differentiation of pancreatic MSCs into adipocytes was also attempted after passage 2 using adipocyte-selective medium; however, after passage 4, pancreatic MSCs could not be differentiated into adipocytes. During adipogenic differentiation, lipid droplets were observed after 7 days by light microscopy (not shown). At 14 days, lipid inclusions were evident by ORO staining (Figure 6a) and differentiated cells expressed FABP4 as measured by IHC (Figure 6b). Pancreatic MSCs not treated with adipogenic medium did not develop lipid droplets and did not express FABP4 (not shown).

Differentiation of pancreatic MSCs into chondrocytes was successful when attempted at the same passages as osteogenesis (passages 2-12) using chondrocyte-selective medium. Differentiated cell pellets harvested at 21 and 28 days of treatment stained with alcian blue (Figure 6c) and expressed aggrecan as measured by IHC (Figure 6d). Undifferentiated tissue did not express aggrecan and cytoplasm did not stain for alcian blue.

\section{In Vitro Differentiation of Pancreatic MSCs into Hepatocytes and Beta-Cells}

Differentiation of pancreatic MSCs into hepatocytes was successful at the passages attempted (passages 2-6). During differentiation, changes in cell morphology were observed after 16 days, or 7 days after the addition of oncostatin-M and dexamethasone. When compared to undifferentiated pancreatic MSCs (Figure 6e), cells acquired a cuboidal morphology (Figure 6f) that persisted throughout differentiation. Differentiated cells harvested at 16 days were human serum albumin immunoreactive (not shown) and cells harvested after 27 days expressed both human serum albumin and GATA 4 demonstrated by IHC (Figure 6g and h). Undifferentiated cells did not express human serum albumin or GATA 4 (not shown). After 27 days of differentiation, cuboidal cells expressed mRNA for both the endoderm-specific gene GATA 4 and liver-specific gene tyrosine aminotransferase (TAT) (Figure 7). At 21 and 24 days only TAT was expressed and prior to 21 days neither gene was expressed (Figure 7). Undifferentiated pancreatic MSCs did not express GATA 4 or TAT, while hepatocytes expressed both GATA 4 and TAT (Figure 7).

The preliminary protocol used in this study to differentiate pancreatic MSCs into beta-cells was somewhat successful when attempted at passage(s) 2-6 $(n=3)$. Changes in cell morphology were not observed during differentiation, but after 9 days of differentiation, the following genes were expressed, as measured by RT-PCR: NeuroD, insulin and glucagon (Figure 8). Following cellular aggregation, in addition to NeuroD, insulin and glucagon, mRNA for ngn3, PDX1 and Pax4 were also expressed (Figure 8) with three patterns of expression observed: (1) ngn3, (2) PDX1 with Pax4 or (3) PDX1 with ngn3. Undifferentiated pancreatic MSCs and cell preparations analyzed after 3 and 6 days of differentiation did not express the above genes (not shown). Although RT-PCR revealed the expression of genes necessary for beta-cell development and function, IHC staining for the gene products was negative (not shown), indicating the mRNA was not translated.

\section{Discussion}

In the present study, we report the in vitro isolation of MSCs from adult human exocrine tissue. We expanded pancreatic MSCs based on their ability to adhere and rapidly divide in the presence of serum, while endocrine and exocrine cell populations declined. These pancreatic MSCs expressed the same cell surface antigens reported to be on MSCs isolated from human $\mathrm{BM}$, adipose and umbilical cord blood. ${ }^{13,16,18,23,24}$ Pancreatic MSCs expressed CD13, CD29, CD44, CD49b, CD54, CD90 and CD105, but did not express CD34, CD45 and CD117. However, since cell surface antigen expression alone 
is not sufficient to characterize MSCs, ${ }^{15}$ we also demonstrated that pancreatic MSCs could be differentiated into mesoderm (osteocytes, adipocytes and chondrocytes). In addition, pancreatic MSCs were shown to be multipotent since they were also capable of deriving endoderm (hepatocytes and beta-cells).

We successfully derived osteocytes from passages 2 to 12 when cells expressed a MSC phenotype. During osteogenesis, tissue was collected at an early time point of 20 days postdifferentiation and later time point of 31 days postdifferentiation. Although osteocalcin expression was detected at both time points during differentiation, a change in the amount of AP activity, mineralization (calcification) and collagen deposition was observed. Only in the earlier time point (20 day) was a large amount of AP activity observed, while only a modest amount calcification and collagen deposition was detected. However, in contrast to the early time point, we repeatedly observed little or no AP activity in the later time points (31 day) that always coincided with a substantial increase in mineralization and collagen deposition. This pattern of AP activity declining as mineralization and collagen deposition increases has been observed when differentiating MSCs isolated from $\mathrm{BM}^{27}$ and is reflective of active osteogenesis and mature compact bone formation despite tissue lifting off during differentiation.

We also successfully differentiated adult pancreatic MSCs into adipocytes and chondrocytes. Although pancreatic MSCs were not capable of adipocyte differentiation after passage 4, osteocyte and chondrocyte differentiation was not affected until changes in cell morphology and phenotype were observed at passage 13. Changes in pancreatic MSC morphology were observed after passage 12, when cells became broad and flat in appearance and ceased to divide. In addition, analysis by flow cytometry demonstrated that cells from passage 13 no longer expressed CD49b or CD13 cell surface antigens. In other studies, MSCs isolated from adult BM cultured in similar conditions were reportedly able to maintain both their phenotype and ability to differentiate throughout multiple passages. ${ }^{15,22,24}$ However, similar studies have reported changes in MSC morphology and phenotype with repeated passages in vitro ${ }^{28-30}$ and have also emphasized the loss of adipogenic potential with higher passage numbers. One study of MSCs isolated from human BM summarized that most MSCs undergoing in vitro expansion lose telomeres with each cell division until reaching a threshold where division ceases and cells assume a senescent phenotype. ${ }^{30}$ Another in vitro study correlated changes in morphology, progressive loss of adipogenic potential and senescent phenotype with an increase in caveolin-1 expression. ${ }^{29}$ In addition, the authors of the latter study concluded that the capacity of adult stem cells could be reflective of donor age, tissue source and biological aging. ${ }^{29}$ Therefore, the phenotypic changes we observed at higher passages and the loss of adipogenic potential are not unique features that can be attributed to pancreatic MSCs or the culture conditions used in our study. Rather, this common characteristic of loss of potential serves to emphasize the limitations faced when expanding adult stem cells.

Although limitations exist when working with adult stem cells, MSCs derived from various somatic tissues are capable of differentiating into mesoderm both in vitro and in vivo, and are considered excellent candidates for cell-based therapy. ${ }^{15,18,21,28,31} \mathrm{In}$ experimental animal models, after myocardial infarction, MSCs when injected at the site of ischemia or systemically delivered migrate to the site of injury, initiate tissue repair and, in some instances, restore function. ${ }^{18,32}$ Therefore, promising therapeutic applications in cardiology are emerging and clinical trials have commenced. ${ }^{33}$ Other clinically relevant cell therapy applications reside with the MSCs ability to cross the mesodermal lineage and derive cells of an endodermal origin. For example, MSCs isolated from BM, umbilical cord blood and adipose are able to differentiate into functional hepatocytes capable of expressing liver-specific genes. ${ }^{19,20,23}$ The same is true for pancreatic MSCs. In this study, we derived cuboidal hepatocytes that expressed liver- and endoderm-specific genes. Similar to other studies, we observed a time-dependent manner in which changes to cell morphology and gene expression were observed. ${ }^{19,23}$ Changes in cell morphology and gene expression were not evident until several days after the addition of oncostatin-M and dexamethasone to the medium. When these same growth factors were omitted, hepatocyte differentiation was not apparent. In addition, IHC demonstrated that these differentiated cells expressed human serum albumin as early as day 16; however, similar to RT-PCR results, GATA 4 was not apparent until after 27 days of differentiation.

Preliminary data also suggest that pancreatic MSCs have the potential to derive beta-cells.

\footnotetext{
Figure 5 Osteogenic differentiation of pancreatic mesenchymal stem cells. Tissue collected after 20 days of treatment with osteoselective medium had abundant alkaline phosphatase (AP) activity stained red (a), some evidence of mineralization stained black by Von Kossa (VK) staining at the left edge of the section (c) and collagen deposition stained green by Gomori's Trichrome stain (e). Immunohistochemical staining for osteocalcin is positive (brown) using the immunoperoxidase method (g). VK stain and osteocalcin are counterstained with hematoxylin (blue). Tissue collected at 31 days of treatment had scant AP activity (b), a significant increase in mineralization stained black (d) compared to images (a and c), overlying the tissue that is composed entirely of collagen, stained green by Trichrome stain (f). Immunohistochemical staining for osteocalcin is still positive (h). Immunoperoxidase stain is brown and hematoxylin counterstain is blue. Scale bar represents $100 \mu \mathrm{m}$.
} 

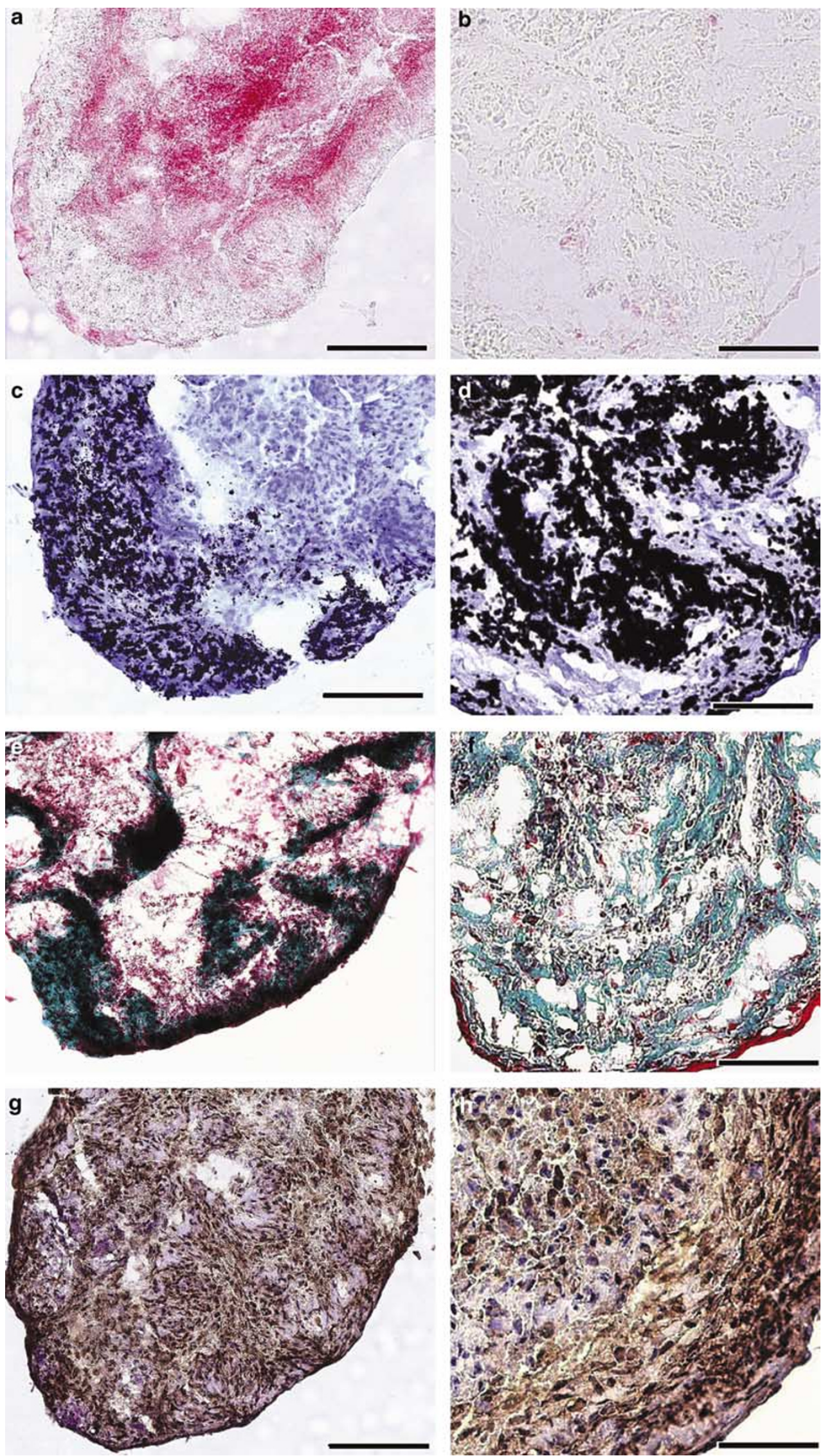

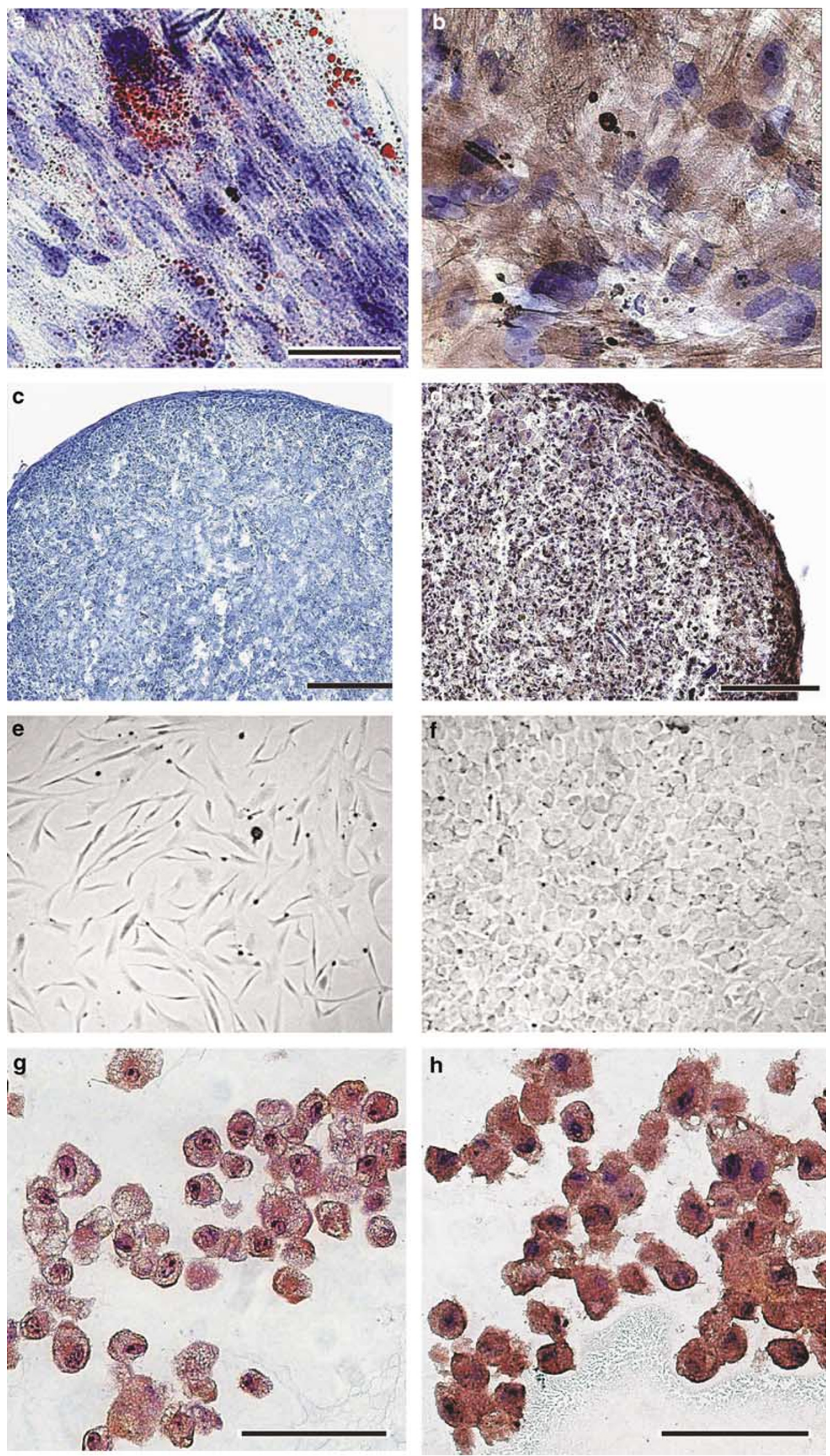


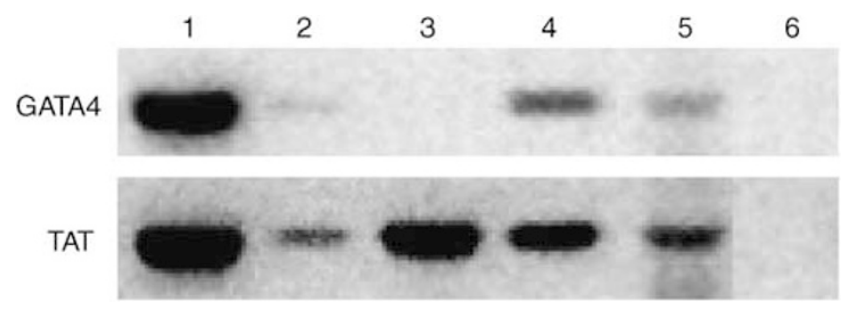

Figure 7 RT-PCR of pancreatic MSCs differentiated into hepatocytes. Message for TAT was detected at 21, 24, 27 and 40 days of hepatocyte differentiation (lanes 2-5). Message for GATA 4 was detected at 27 and 40 days of differentiation (lanes 4 and 5). Undifferentiated pancreatic MSCs did not express message for either gene (lane 6) and human hepatocytes expressed both TAT and GATA 4 (lane 1).

Analyses of preaggregated tissue during differentiation demonstrated that cells could express islet cell mRNA previously seen in the initial ductal cell aggregates used to expand MSCs. In addition, further differentiation by aggregating in the presence of growth factors resulted in the expression of the transcription factors $P D X 1, P a x 4$ and ngn3, where Pax4 and ngn3 were not expressed in the initial ductal cell aggregates. Although multiple patterns of gene expression were observed in these initial experiments, which could be due to donor variability, such as age, and IHC staining was negative, this preliminary data suggest the potential for deriving functional beta-cells and warrants further investigation. For example, infection of mouse and human duct cell lines and/or isolated duct epithelial cells $^{34,35}$ with an ngn3-expressing adenovirus or plasmid encoding $P D X 1^{36}$ resulted in the expression of genes critical to islet differentiation and insulin expression that include Pax4 and NeuroD., ${ }^{34,35}$ Therefore, the fact that we were able to induce the expression of these genes without adenoviral infection suggests that with further in vitro and possible in vivo manipulation, pancreatic MSCs could be induced to express functional islet proteins.

Since there is an inherent shortage of donor islets for the transplantation of patients with type I diabetes, our long-term goal is to identify and expand the 'islet/beta-cell progenitor' hypothesized to reside within the pancreatic ductal epithelium. Therefore, we are continuing to explore the multipotency of the pancreatic MSC and assess its complete potential. However, if, as postulated recently by Gershengorn et $a l^{37}$ and Linning et $a l^{38}$ that fibroblast-like cells residing within the pan-

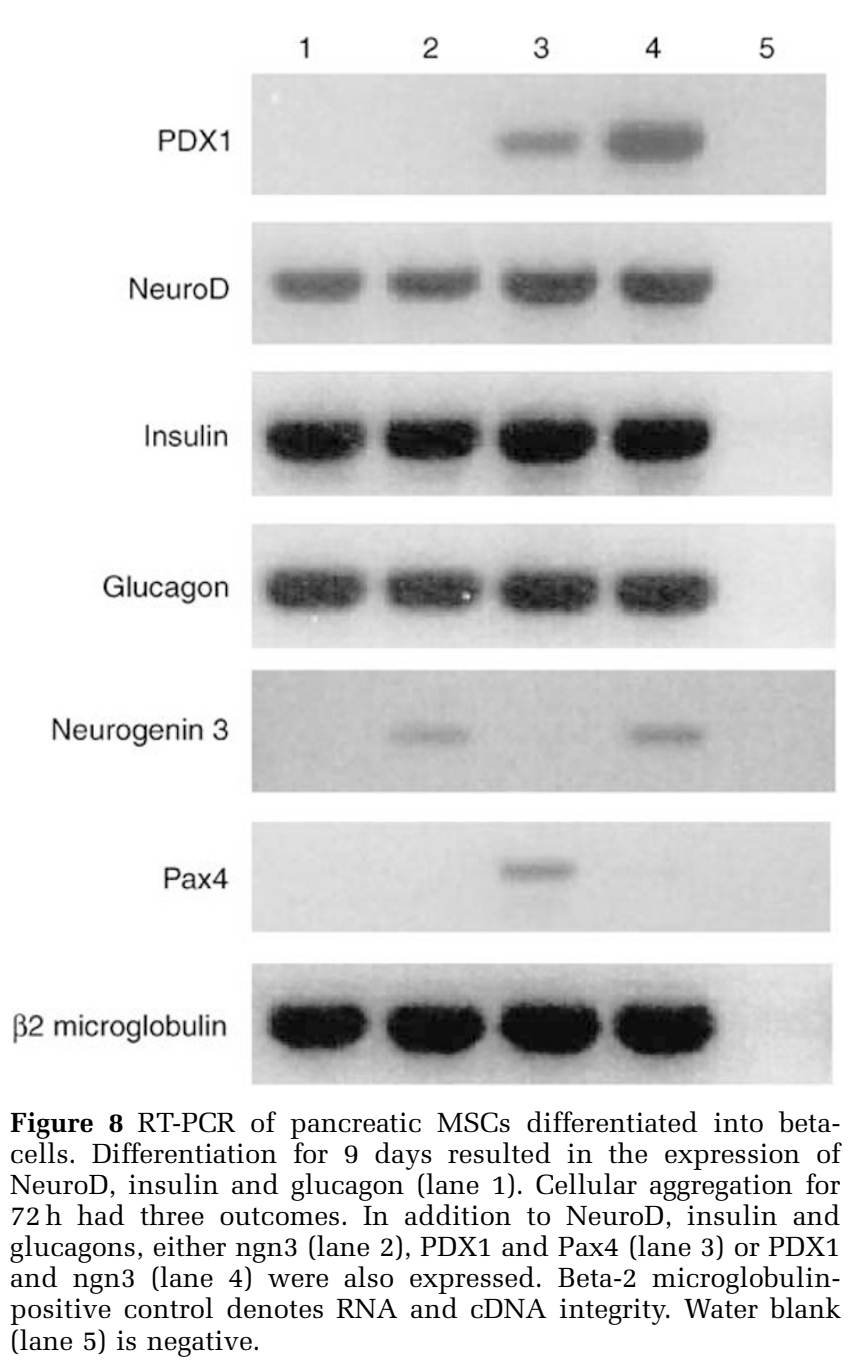

creas are multipotent and capable of reversible endoderm to mesoderm transitions, perhaps pancreatic MSCs could represent an abundant source of islet progenitors and potentially derive sufficient numbers of insulin-producing cells required for transplantation.

\section{Acknowledgements}

Funding for this project was provided by grants from the Canadian Institutes of Health Research (CIHR) Grant FRN-8030, Alberta Heritage Foundation for Medical Research (AHFMR), Juvenile Diabetes

Figure 6 Representative image of pancreatic MSCs differentiated into adipocytes (a, b), chondrocytes (c, d) and hepatocytes (e-h). ORO staining detects lipid droplets (a) and immunohistochemical staining for FABP4 (brown) is positive (b). Lipid droplets are red, positive immunoperoxidase stain is brown and hematoxylin counterstain is blue. Chondrocyte pellet is positive for alcian blue stain (c) and immunohistochemical staining for aggrecan is positive (d). Positive immunoperoxidase stain is brown and hematoxylin counterstain is blue. Scale bar represents $50 \mu \mathrm{m}$. Hepatogenic differentiation of pancreatic MSCs (e-h). Phase contrast demonstrates that after 16 days, pancreatic MSCs lose their fibroblast-like morphology (e) and assume a cuboidal morphology (f). Magnification $\times 40$. Immunohistochemical staining for human serum albumin (g) and GATA 4 (h) is positive in cuboidal cells harvested after 27 days of differentiation. Positive immunoperoxidase stain is brown and hematoxylin counterstain is blue. Scale bar represents $50 \mu \mathrm{m}$. 
Research Foundation (JDRF) and the Stem Cell Network. JMD is a recipient of a Postdoctoral Fellowship from the AHFMR and CIHR. JRTL is a scholar of the AHFMR. AMJS is a clinical investigator of the AHFMR and holds the Clinical Research Chair in Transplantation (CIHR/Wyeth). GSK is a senior scholar of the AHFMR, and a recipient of a Career Development Award from the JDRF. We would like to thank Alana Eshpeter and Serena Sah for technical assistance and data collection. We also thank the staff of the Clinical Islet Laboratory (University of Alberta) as well as the HOPE program.

\section{References}

1 Shapiro AM, Lakey JR, Ryan EA, et al. Islet transplantation in seven patients with type 1 diabetes mellitus using a glucocorticoid-free immunosuppressive regimen. N Engl J Med 2000;343:230-238.

2 Ryan EA, Lakey JR, Rajotte RV, et al. Clinical outcomes and insulin secretion after islet transplantation with the Edmonton protocol. Diabetes 2001;50:710-719.

3 Zulewski H, Abraham EJ, Gerlach MJ, et al. Multipotential nestin-positive stem cells isolated from adult pancreatic islets differentiate ex vivo into pancreatic endocrine, exocrine, and hepatic phenotypes. Diabetes 2001;50:521-533.

4 Bonner-Weir S, Taneja M, Weir GC, et al. In vitro cultivation of human islets from expanded ductal tissue. Proc Natl Acad Sci USA 2000;97:7999-8004.

5 Petropavlovskaia M, Rosenberg L. Identification and characterization of small cells in the adult pancreas: potential progenitor cells? Cell Tissue Res 2002;310: 51-58.

6 Gao R, Ustinov J, Pulkkinen MA, et al. Characterization of endocrine progenitor cells and critical factors for their differentiation in human adult pancreatic cell culture. Diabetes 2003;52:2007-2015.

7 Rooman I, Lardon J, Bouwens L. Gastrin stimulates beta-cell neogenesis and increases islet mass from transdifferentiated but not from normal exocrine pancreas tissue. Diabetes 2002;51:686-690.

8 Wang RN, Kloppel G, Bouwens L. Duct- to islet-cell differentiation and islet growth in the pancreas of ductligated adult rats. Diabetologia 1995;38:1405-1411.

9 Street CN, Lakey JR, Shapiro AM, et al. Islet graft assessment in the Edmonton protocol: implications for predicting long-term clinical outcome. Diabetes 2004;53:3107-3114.

10 Abraham EJ, Leech CA, Lin JC, et al. Insulinotropic hormone glucagon-like peptide-1 differentiation of human pancreatic islet-derived progenitor cells into insulin-producing cells. Endocrinology 2002;143: 3152-3161.

11 Street CN, Lakey JR, Seeberger K, et al. Heterogenous expression of nestin in human pancreatic tissue precludes its use as an islet precursor marker. J Endocrinol 2004;180:213-225.

12 Street CN, Lakey JR, Rajotte RV, et al. Enriched human pancreatic ductal cultures obtained from selective death of acinar cells express pancreatic and duodenal homeobox gene-1 age-dependently. Rev Diabetic Stud 2004;1:66-79 (Society for Biomedical Diabetes Research: Duisburg, Germany).
13 Zuk PA, Zhu M, Ashjian P, et al. Human adipose tissue is a source of multipotent stem cells. Mol Biol Cell 2002;13:4279-4295.

14 De Ugarte DA, Alfonso Z, Zuk PA, et al. Differential expression of stem cell mobilization-associated molecules on multi-lineage cells from adipose tissue and bone marrow. Immunol Lett 2003;89:267-270.

15 Javazon EH, Beggs KJ, Flake AW. Mesenchymal stem cells: paradoxes of passaging. Exp Hematol 2004;32: 414-425.

16 Pittenger MF, Mackay AM, Beck SC, et al. Multilineage potential of adult human mesenchymal stem cells. Science 1999;284:143-147.

17 Zuk PA, Zhu M, Mizuno H, et al. Multilineage cells from human adipose tissue: implications for cell-based therapies. Tissue Eng 2001;7:211-228.

18 Pittenger MF, Martin BJ. Mesenchymal stem cells and their potential as cardiac therapeutics. Circ Res 2004; 95:9-20.

19 Lee KD, Kuo TK, Whang-Peng J, et al. In vitro hepatic differentiation of human mesenchymal stem cells. Hepatology 2004;40:1275-1284.

20 Seo MJ, Suh SY, Bae YC, et al. Differentiation of human adipose stromal cells into hepatic lineage in vitro and in vivo. Biochem Biophys Res Commun 2005; 328:258-264.

21 Barbash IM, Chouraqui P, Baron J, et al. Systemic delivery of bone marrow-derived mesenchymal stem cells to the infarcted myocardium: feasibility, cell migration, and body distribution. Circulation 2003; 108:863-868.

22 Sordi V, Malosio ML, Marchesi F, et al. Bone marrow mesenchymal stem cells express a restricted set of functionally active chemokine receptors capable of promoting migration to pancreatic islets. Blood 2005; 105:419-427.

23 Lee OK, Kuo TK, Chen WM, et al. Isolation of multipotent mesenchymal stem cells from umbilical cord blood. Blood 2004;103:1669-1675.

24 Reyes M, Lund T, Lenvik T, et al. Purification and ex vivo expansion of postnatal human marrow mesodermal progenitor cells. Blood 2001;98:2615-2625.

25 McManus JFA, Mowry R. Staining Methods Histological and Histochemical. Paul B Hoeber: New York, 1960.

26 Lee RH, Kim B, Choi I, et al. Characterization and expression analysis of mesenchymal stem cells from human bone marrow and adipose tissue. Cell Physiol Biochem 2004;14:311-324.

27 Jaiswal N, Haynesworth SE, Caplan AI, et al. Osteogenic differentiation of purified, culture-expanded human mesenchymal stem cells in vitro. J Cell Biochem 1997;64:295-312.

28 Oswald J, Boxberger S, Jorgensen B, et al. Mesenchymal stem cells can be differentiated into endothelial cells in vitro. Stem Cells 2004;22:377-384.

29 Park JS, Kim HY, Kim HW, et al. Increased caveolin-1, a cause for the declined adipogenic potential of senescent human mesenchymal stem cells. Mech Ageing Dev 2005;126:551-559.

30 Baxter MA, Wynn RF, Jowitt SN, et al. Study of telomere length reveals rapid aging of human marrow stromal cells following in vitro expansion. Stem Cells 2004;22:675-682.

31 Tholpady SS, Katz AJ, Ogle RC. Mesenchymal stem cells from rat visceral fat exhibit multipotential differentiation in vitro. Anat Rec 2003;272A:398-402. 
32 Shake JG, Gruber PJ, Baumgartner WA, et al. Mesenchymal stem cell implantation in a swine myocardial infarct model: engraftment and functional effects. Ann Thorac Surg 2002;73:1919-1925.

33 Chen SL, Fang WW, Ye F, et al. Effect on left ventricular function of intracoronary transplantation of autologous bone marrow mesenchymal stem cell in patients with acute myocardial infarction. Am J Cardiol 2004;94:92-95.

34 Gasa R, Mrejen C, Leachman N, et al. Proendocrine genes coordinate the pancreatic islet differentiation program in vitro. Proc Natl Acad Sci USA 2004;101: 13245-13250.

35 Heremans Y, Van De Casteele M, in't Veld P, et al. Recapitulation of embryonic neuroendocrine differen- tiation in adult human pancreatic duct cells expressing neurogenin 3. J Cell Biol 2002;159:303-311.

36 Zhao M, Amiel SA, Christie MR, et al. Insulinproducing cells derived from human pancreatic nonendocrine cell cultures reverse streptozotocin-induced hyperglycaemia in mice. Diabetologia 2005;48:20512061.

37 Gershengorn MC, Hardikar AA, Wei C, et al. Epithelialto-mesenchymal transition generates proliferative human islet precursor cells. Science 2004;306:22612264.

38 Linning KD, Tai MH, Madhukar BV, et al. Redoxmediated enrichment of self-renewing adult human pancreatic cells that possess endocrine differentiation potential. Pancreas 2004;29:e64-e76. 